\title{
Padé approximants for the acoustical properties of rigid frame porous media with pore size distributions
}

Article

Published Version

Horoshenkov, K. V., Attenborough, K. and Chandler-Wilde, S. N. (1998) Padé approximants for the acoustical properties of rigid frame porous media with pore size distributions. Journal of the Acoustical Society of America, 104 (3). pp. 1198-1209. ISSN 0001-4966 doi: https://doi.org/10.1121/1.424328 Available at https://centaur.reading.ac.uk/32656/

It is advisable to refer to the publisher's version if you intend to cite from the work. See Guidance on citing.

Published version at: http://dx.doi.org/10.1121/1.424328

To link to this article DOI: http://dx.doi.org/10.1121/1.424328

Publisher: Acoustical Society of America

All outputs in CentAUR are protected by Intellectual Property Rights law, including copyright law. Copyright and IPR is retained by the creators or other copyright holders. Terms and conditions for use of this material are defined in the End User Agreement.

www.reading.ac.uk/centaur 
Central Archive at the University of Reading

Reading's research outputs online 


\title{
Padé approximants for the acoustical properties of rigid frame porous media with pore size distributions
}

\author{
K. V. Horoshenkov \\ Department of Civil and Environmental Engineering, University of Bradford, Bradford BD7 1DP, \\ United Kingdom \\ Keith Attenborough \\ Faculty of Technology, Open University, Milton Keynes MK7 6AA, United Kingdom \\ S. N. Chandler-Wilde \\ Department of Mathematics and Statistics, Brunel Unversity, Uxbridge UB8 3PH, United Kingdom
}

(Received 21 July 1997; accepted for publication 5 June 1998)

\begin{abstract}
Expressions for the viscosity correction function, and hence bulk complex impedance, density, compressibility, and propagation constant, are obtained for a rigid frame porous medium whose pores are prismatic with fixed cross-sectional shape, but of variable pore size distribution. The lowand high-frequency behavior of the viscosity correction function is derived for the particular case of a log-normal pore size distribution, in terms of coefficients which can, in general, be computed numerically, and are given here explicitly for the particular cases of pores of equilateral triangular, circular, and slitlike cross-section. Simple approximate formulae, based on two-point Padé approximants for the viscosity correction function are obtained, which avoid a requirement for numerical integration or evaluation of special functions, and their accuracy is illustrated and investigated for the three pore shapes already mentioned. (c) 1998 Acoustical Society of America. [S0001-4966(98)04009-0]
\end{abstract}

PACS numbers: 43.20.Bi, 43.20.Mv [DAC]

\section{INTRODUCTION}

There have been several studies related to the theoretical description of porous granular materials that extend and augment the original analysis by Biot. ${ }^{1}$ Two of the most interesting developments have been perpetrated by Stinson ${ }^{2}$ and Yamamoto and Turgut $^{3}$ and allow for arbitrary pore shape and distributions of pore sizes, respectively. As a consequence of Stinson's work, exact analytical results for cylindrical, slitlike, triangular, and rectangular pore shapes are available. Yamamoto and Turgut concentrated on the case of a log-normal pore size distribution in sedimentary materials. Attenborough ${ }^{4}$ has shown that, of the possible pore characteristics, the presence of a variable distribution of pore sizes potentially has greater effect than change in pore shape. This is of particular practical interest since pore size distribution is routinely measurable. A different type of development, but of relevance to further progress in routine calculations of acoustical properties of rigid-framed porous materials is the use of Padé approximants. These have been developed for structures consisting of uniform pores in Ref. 5. In this paper these various contributions are combined. Padé approximants for the acoustical properties of media with size distributions of variously shaped pores are derived. It is anticipated that these approximations offer a practical and efficient alternative to increasingly sophisticated models (see, for example, Ref. 4) for sound propagation in a medium with pore size distribution.

In the first section an argument is presented to justify the use of the viscosity correction function of Yamamoto and Turgut $^{3}$ in computing the bulk acoustic properties of rigidframe porous media. An expression is derived for the viscos- ity correction function in terms of a single integral involving the pore size probability density function and a single function of a complex variable, $\bar{\psi}(z)$, specified in terms of the pore shape. This expression is simplified further for the important case of a log-normal pore size distribution in Sec. II. Low- and high-frequency approximations to the viscosity correction function are obtained in the second section, using results on approximation of a class of integrals in the Appendix. These approximations are used to derive two-point Padé approximants for the viscosity correction function in Sec. IV, and the accuracy of these simple approximants in calculating the acoustic characteristics such as relative admittance and complex wavenumber is explored.

\section{THE VISCOSITY CORRECTION FUNCTION}

The majority of porous materials are composed of pores of variable shape and size which obey a distinctive statistical distribution. For these materials we can only refer to the values of the dynamic density and complex compressibility which have been averaged over a range of realistic pore sizes identified in the material. The same argument is applicable to acoustic quantities such as the acoustic admittance and propagation constant.

To derive the general form for the viscosity correction function we consider a sample of a bulk rigid-frame porous material which is pierced through by normal to the surface prismatic (i.e., uniform with respect to cross-sectional geometry) straight pores of various sizes but the same crosssectional shape. Let us characterize the different sizes of pore by measurement of some linear dimension $s$ and let $E(s)$ denote the pore size distribution function, that is, $E(s)$ is the 
fraction of the total pore volume consisting of pores of size not exceeding $s$. Then $e(s)=d E(s) / d s$ is the corresponding probability density function.

Within a typical pore of size $s$ the velocity component $u$ in the direction of the pore axis (the $x_{3}$-direction) satisfies, for harmonic $\left(e^{-i \omega t}\right)$ time dependence, to a good approximation the force equation ${ }^{2}$

$$
i \omega \rho_{0} u+\mu \Delta u=\frac{d p}{d x_{3}} .
$$

In this equation $\rho_{0}$ is the equilibrium fluid density, $\mu$ the dynamic viscosity, a Cartesian coordinate system $O x_{1} x_{2} x_{3}$ has been adopted, $\Delta=\partial^{2} / \partial x_{1}^{2}+\partial^{2} / \partial x_{2}^{2}$, and $p$ is the pressure in the fluid which depends, to a good approximation, only on $x_{3}$. The velocity $u$ vanishes on the sides of the pore.

Similarly, ${ }^{2}$ the excess temperature $T$ satisfies that

$$
\kappa \Delta T+i \rho_{0} \omega C_{p} T=i \omega p,
$$

where $\kappa$ is the thermal conductivity of the fluid and $C_{p}$ its specific heat at constant pressure. The excess temperature is assumed to vanish on the sides of the pore.

We introduce an auxiliary function $\psi=\psi\left(x_{1}, x_{2} ; w\right)$ which is defined by a boundary value problem which it satisfies within the pore cross-section of a unit size pore of dimension $s=1$. This two-dimensional boundary value problem consists of the equation

$$
\Delta \psi-w^{2} \psi=1
$$

within the pore cross-section, where $w$ is a given constant, and the boundary condition $\psi=0$ around the edge of the section. The form of the function $\psi$ depends on the value of the real or complex parameter $w$. We assume that $-\pi / 2$ $<\arg w<\pi / 2$ in which case $\psi$ is uniquely determined by these imposed conditions.

In terms of this auxiliary function $\psi$ it is easy to see, by substitution, that the solutions of (1) and (2) which vanish on the walls of the pore are

$$
u=\frac{s^{2}}{\mu} \frac{d p}{d z} \psi\left(x_{1} / s, x_{2} / s ; \sqrt{-i} \lambda\right)
$$

and

$$
T=\frac{i \omega s^{2}}{\kappa} p \psi\left(x_{1} / s, x_{2} / s ; \sqrt{-i} N_{\mathrm{pr}}^{1 / 2} \lambda\right),
$$

where $N_{\mathrm{pr}}$ is the Prandtl number and

$$
\lambda=\left(\rho_{0} \omega / \mu\right)^{1 / 2} s .
$$

For any fluid property $\phi$ let $\bar{\phi}$ denote the mean value over the cross-section and $\langle\phi\rangle$ denote the mean value of $\bar{\phi}$ over all pore sizes, i.e.,

$$
\langle\phi\rangle=\int_{0}^{\infty} e(s) \bar{\phi}(s) d s .
$$

Then, if we assume that there are sufficient interconnections between the pores of different sizes so that the dependence of $p$ on $x_{3}$ is independent of the pore size $s$, it follows from (1) that

$$
i \omega \rho_{0}\langle u\rangle+\mu\langle\Delta u\rangle=\frac{d p}{d x_{3}},
$$

an equation which we can write as

$$
i \omega \rho_{x}(\omega)\langle u\rangle=\frac{d p}{d x_{3}},
$$

where $\rho_{x}(\omega)$ is an effective complex density averaged over the pore size given by

$$
\rho_{x}(\omega)=\rho_{0}+\frac{\mu\langle\Delta u\rangle}{i \omega\langle u\rangle} .
$$

In physical terms $\langle u\rangle$ is the mean velocity over all pores, i.e., the total volume flux through the pores divided by the total pore cross-sectional area. By Gauss' theorem,

$$
\mu \overline{\Delta u}=\frac{\mu}{A} \int_{P} \frac{\partial u}{\partial n} d s=\frac{1}{A} \int_{P} \tau d s,
$$

where $P$ is the perimeter of the cross-section and $\tau$ the shear stress. Thus $\mu\langle\Delta u\rangle=\langle\tau\rangle$, where $\langle\tau\rangle$ denotes the total shear force in the $x_{3}$-direction per unit thickness of the sample, per unit cross-sectional area, so that

$$
\rho_{x}(\omega)=\rho_{0}+\frac{1}{i \omega} \frac{\langle\tau\rangle}{\langle u\rangle} .
$$

In terms of the auxiliary function $\psi$ we easily see that

$$
\bar{u}=\frac{s^{2}}{\mu} \frac{d p}{d x_{3}} \bar{\psi}, \quad \overline{\Delta u}=\frac{1}{\mu} \frac{d p}{d x_{3}} \overline{\Delta \psi},
$$

where $\bar{\psi}=\bar{\psi}(\sqrt{-i} \lambda)$ and $\overline{\Delta \psi}=\overline{\Delta \psi}(\sqrt{-i} \lambda)$ are the mean values over the cross-section of the pore of size $s=1$ of $\psi$ and $\Delta \psi$, respectively, when $w=\sqrt{-i} \lambda$. Clearly

$$
\rho_{x}(\omega)=\rho_{0}-\frac{R_{x}}{i \omega} F(\omega),
$$

where

$$
R_{x}=-\lim _{\omega \rightarrow 0} \frac{\langle\tau(\omega)\rangle}{\langle u(\omega)\rangle}
$$

is an effective DC flow resistivity averaged over the pores, and

$$
F(\omega)=\frac{\langle\tau(\omega)\rangle}{\langle u(\omega)\rangle}\left[\lim _{\omega \rightarrow 0} \frac{\langle\tau(\omega)\rangle}{\langle u(\omega)\rangle}\right]^{-1}
$$

is the viscosity correction function as utilised by Yamamoto and Turgut $^{3}$ and Attenborough. ${ }^{4}$ In terms of the auxiliary function $\psi$ we have that, since $i \lambda^{2} \bar{\psi}+\overline{\Delta \psi}=1$ from (3),

$$
F(\omega)=-\frac{i \omega \rho_{0} I(\omega)}{R_{x}(1-I(\omega))},
$$

where

$$
\begin{aligned}
I(\omega) & =\int_{0}^{\infty} e(s) \overline{\Delta \psi}(\sqrt{-i} \lambda) d s \\
& =1-\frac{i \omega \rho_{0}}{\mu} \int_{0}^{\infty} s^{2} e(s) \bar{\psi}(\sqrt{-i} \lambda) d s .
\end{aligned}
$$


In the remainder of this paper we concentrate on the problem of evaluating the viscosity correction function $F(\omega)$. Once $F(\omega)$ is known we can evaluate the dynamic density $\rho_{x}(\omega)$. Further, similarly to the definition of (9), we obtain from (2) that

$$
\theta(\omega)\langle T\rangle=p
$$

with

$$
\theta(\omega)=\frac{C_{p} \rho_{0}}{1-I\left(N_{\mathrm{pr}} \omega\right)}=C_{p} \rho_{x}\left(N_{\mathrm{pr}} \omega\right) .
$$

From (9) and (19), the equation of continuity, and the ideal gas law, we obtain that $p$ satisfies the usual onedimensional Helmholtz equation with complex wave number $k_{x}(\omega)$ as in Ref. 2, but with single pore size values replaced by averaged values. Precisely

$$
\frac{d^{2} p}{d x_{3}^{2}}+k_{x}^{2}(\omega) p=0
$$

with

$$
k_{x}^{2}(\omega)=\omega^{2} \rho_{x}(\omega) C_{x}(\omega)
$$

and the complex compressibility, $C_{x}(\omega)$, given by

$$
C_{x}(\omega)=\frac{1}{P_{0}}-\frac{1}{T_{0} \theta(\omega)}=\frac{1}{\gamma P_{0}}\left(\gamma-\frac{\rho_{0}(\gamma-1)}{\rho_{x}\left(N_{\mathrm{pr}} \omega\right)}\right) .
$$

Finally, we include the effects of tortuosity and of the sample porosity $\Omega$. Relaxing the condition that the pores be normal to the sample surface, let $q \geqslant 1$ be the ratio of pore length to sample thickness so that $q^{2}$ is the tortuosity. Then $\langle v\rangle=\Omega\langle u\rangle / q$ is the bulk velocity, the total volume flux divided by the sample cross-sectional area. Equation (9) holds with $\langle u\rangle$ replaced by $\langle v\rangle$ provided we replace $\rho_{x}(\omega)$ by a bulk medium dynamic complex density $\rho_{b}(\omega)$ $=\left(q^{2} / \Omega\right) \rho_{x}(\omega)$. Thus

$$
\rho_{b}(\omega)=\frac{q^{2}}{\Omega}\left(\rho_{0}-\frac{\Omega R_{b}}{i \omega q^{2}} F(\omega)\right),
$$

where we have introduced the bulk flow resistivity, $R_{b}$ $=q^{2} R_{x} / \Omega$. Equation (21) holds with $x_{3}$ replaced by $q x_{3}$, alternatively with $k_{x}(\omega)$ replaced by the bulk medium wave number $k_{b}(\omega)=q k_{x}(\omega)$. Then Eq. (22) holds with $\rho_{x}, k_{x}, C_{x}$ replaced by their bulk medium values, i.e.,

$$
k_{b}^{2}(\omega)=\omega^{2} \rho_{b}(\omega) C_{b}(\omega),
$$

provided we define the bulk medium complex compressibility by $C_{b}(\omega)=\Omega C_{x}(\omega)$. Finally the complex impedance of the medium is

$$
Z_{b}(\omega)=p /\langle v\rangle=\omega \rho_{b}(\omega) / k_{b}(\omega)=\sqrt{\rho_{b}(\omega) / C_{b}(\omega)},
$$

so that the bulk medium admittance relative to that of the fluid medium is

$$
\beta_{b}(\omega)=\rho_{0} k_{b}(\omega) /\left(k \rho_{b}(\omega)\right)=(q / \Omega) \beta_{x}(\omega),
$$

where $k$ is the wave number in the fluid medium and

$$
\beta_{x}(\omega)=\rho_{0} k_{x}(\omega) /\left(k \rho_{x}(\omega)\right) .
$$

\section{LOG-NORMAL PORE SIZE DISTRIBUTION}

The expressions presented in the previous section are only practical if the pore size distribution $e(s)$ in the material is known. Experimental values for the statistical distribution $e(s)$, which can be measured by either the water suction method $^{6}$ or the mercury injection technique ${ }^{7}$ or recovered from acoustical experiments, ${ }^{8}$ are normally replaced in Eq. (18) with an explicit expression which provides a good fit to the experimental data.

It has been found from numerous experiments in geophysics and in outdoor noise propagation that in many granular materials ${ }^{4,7}$ the real pore size distribution $e(s)$ can be closely approximated by a log-normal statistical distribution.

In the $\log$-normal statistical distribution $\phi=-\log _{2} s$ is normally distributed with mean $\bar{\phi}=-\log _{2} \bar{s}$, where $\bar{s}$ is the median pore size, and standard deviation $\sigma$. Thus, where $G(\phi)$ is the distribution function of $\phi$,

$$
E(s)=1-G(\phi)
$$

so that

$$
e(s)=E^{\prime}(s)=-g(\phi) \frac{d \phi}{d s},
$$

where $g(\phi)=G^{\prime}(\phi)$ is the probability density function of $\phi$, i.e.,

$$
g(\phi)=\frac{1}{\sqrt{2 \pi} \sigma} \exp \left(-\frac{(\phi-\bar{\phi})^{2}}{2 \sigma^{2}}\right) .
$$

The standard deviation $\sigma$ is dimensionless and is a measure of the deviation of $s$ about its median value in relative terms. Precisely, for any $\alpha>0$,

$$
\operatorname{Pr}\left(\frac{1}{2^{\sigma \alpha}} \leqslant \frac{s}{\bar{s}} \leqslant 2^{\sigma \alpha}\right)=\operatorname{Pr}\left(\left|\frac{\phi-\bar{\phi}}{\sigma}\right| \leqslant \alpha\right)=2 \Phi(\alpha)-1,
$$

where

$$
\Phi(\alpha)=\frac{1}{\sqrt{2 \pi}} \int_{-\infty}^{\alpha} e^{-y^{2} / 2} d y
$$

is the distribution function of the standard normal distribution.

Recall that the viscosity correction function is given in terms of $I(\omega)$ by (17). For a log-normal distribution, substituting $s=2^{-\phi}$ in (18) and noting (30), we obtain that

$$
I(\omega)=1-\frac{i \omega \rho_{0}}{\mu} \int_{-\infty}^{+\infty} 2^{-2 \phi} g(\phi) \bar{\psi}(\sqrt{-i} \lambda) d \phi .
$$

Defining

$$
\bar{\lambda}=\left(\frac{\rho_{0} \omega}{\mu}\right)^{1 / 2} \bar{s}
$$

to be the median value of $\lambda$, noting (6) and (31), and substituting $\phi=\bar{\phi}+\sigma t$, we see that, for a log-normal distribution, $I(\omega)$ depends only on the dimensionless parameter combinations $\bar{\lambda}$ and $\sigma$ : precisely, 

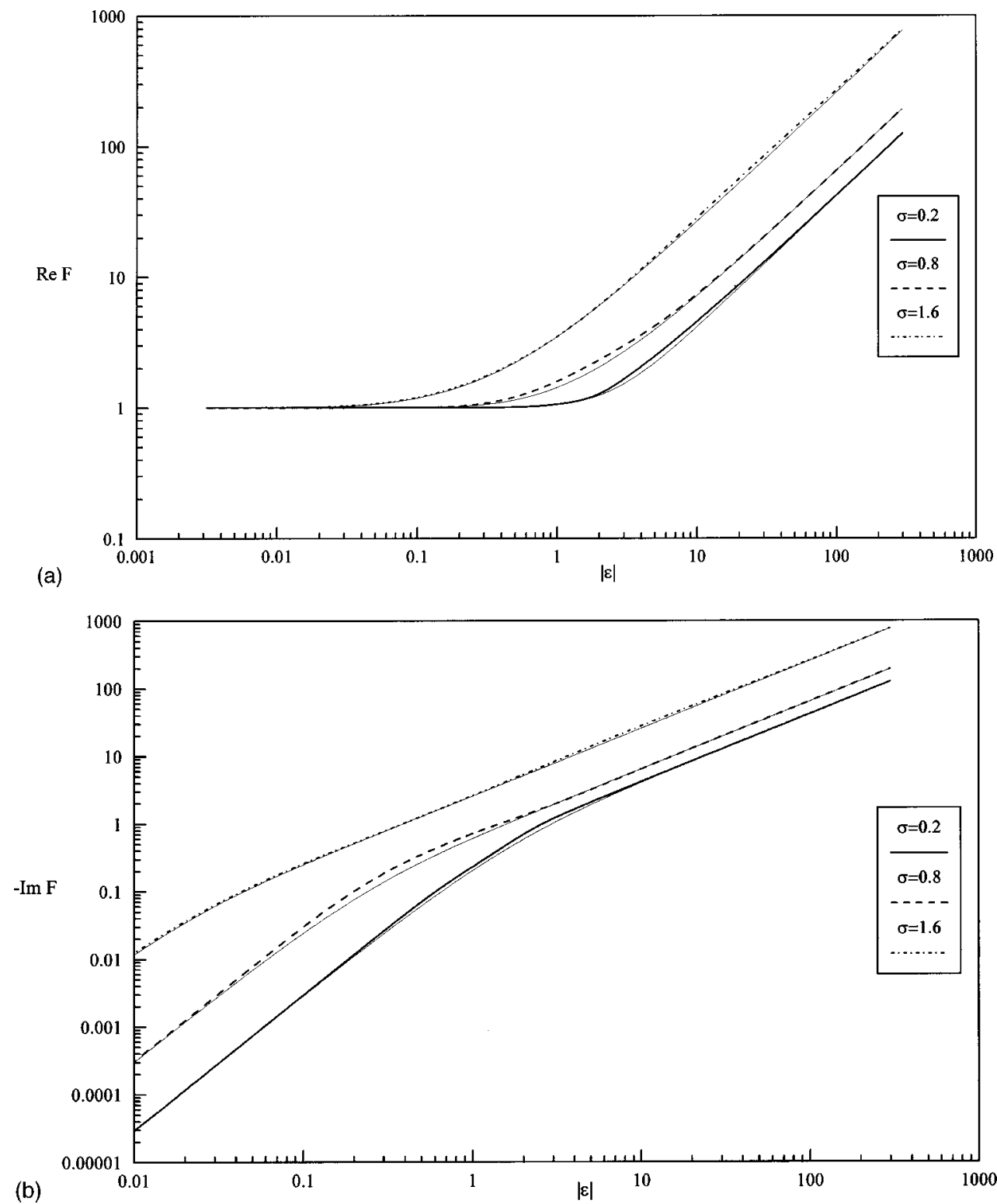

FIG. 1. (a) The real part of the viscosity correction function and its Padé approximant (thin solid lines) in the case of a material with slitlike pores. (b) The imaginary part of the viscosity correction function and its Padé approximant (thin solid lines) in the case of a material with slitlike pores.

$$
\begin{aligned}
I(\omega) & =1-\frac{i \bar{\lambda}^{2}}{\sqrt{2 \pi}} \int_{-\infty}^{+\infty} 2^{-2 \sigma t} e^{-t^{2} / 2} \bar{\psi}\left(\sqrt{-i} \bar{\lambda} 2^{-\sigma t}\right) d t \\
& =\frac{1}{\sqrt{2 \pi}} \int_{-\infty}^{+\infty} \chi\left(\sqrt{-i} \bar{\lambda} 2^{-\sigma t}\right) e^{-t^{2} / 2} d t,
\end{aligned}
$$

where the function $\chi$, which depends only on the pore shape, is defined by

$$
\chi(z)=1+z^{2} \bar{\psi}(z)
$$

for $-\pi / 2<\arg z<\pi / 2$. From (14) to (17) it can be seen that

$$
I(\omega)=1-\frac{\rho_{0}}{\rho_{x}(\omega)}
$$

and, in particular, this holds in the case $\sigma=0$ of fixed pore size distribution $s=\bar{s}$. In this case (34) reduces to

$$
I(\omega)=\chi(\sqrt{-i} \bar{\lambda}) .
$$

It is probably easiest to extract from the literature the form of the function $\chi$ for various pore shapes using these last two equations (36) and (37). Using these equations, from Stinson and Champoux, ${ }^{2}$ for pores which are infinite rectangular slits of half-width $s$, we obtain that

$$
\chi(z)=\frac{\tanh z}{z} .
$$

For circular pores of radius $s$, we find

$$
\chi(z)=\frac{2 I_{1}(z)}{z I_{0}(z)},
$$

where $I_{0}$ and $I_{1}$ are the modified Bessel functions of order one and zero, respectively. For equilateral triangular pores, with $s$ the length of the triangle side,

$$
\chi(z)=3\left(\frac{\operatorname{coth}(\sqrt{3} / 4 z)}{\sqrt{3} / 4}-\frac{16}{3 z^{2}}\right) .
$$



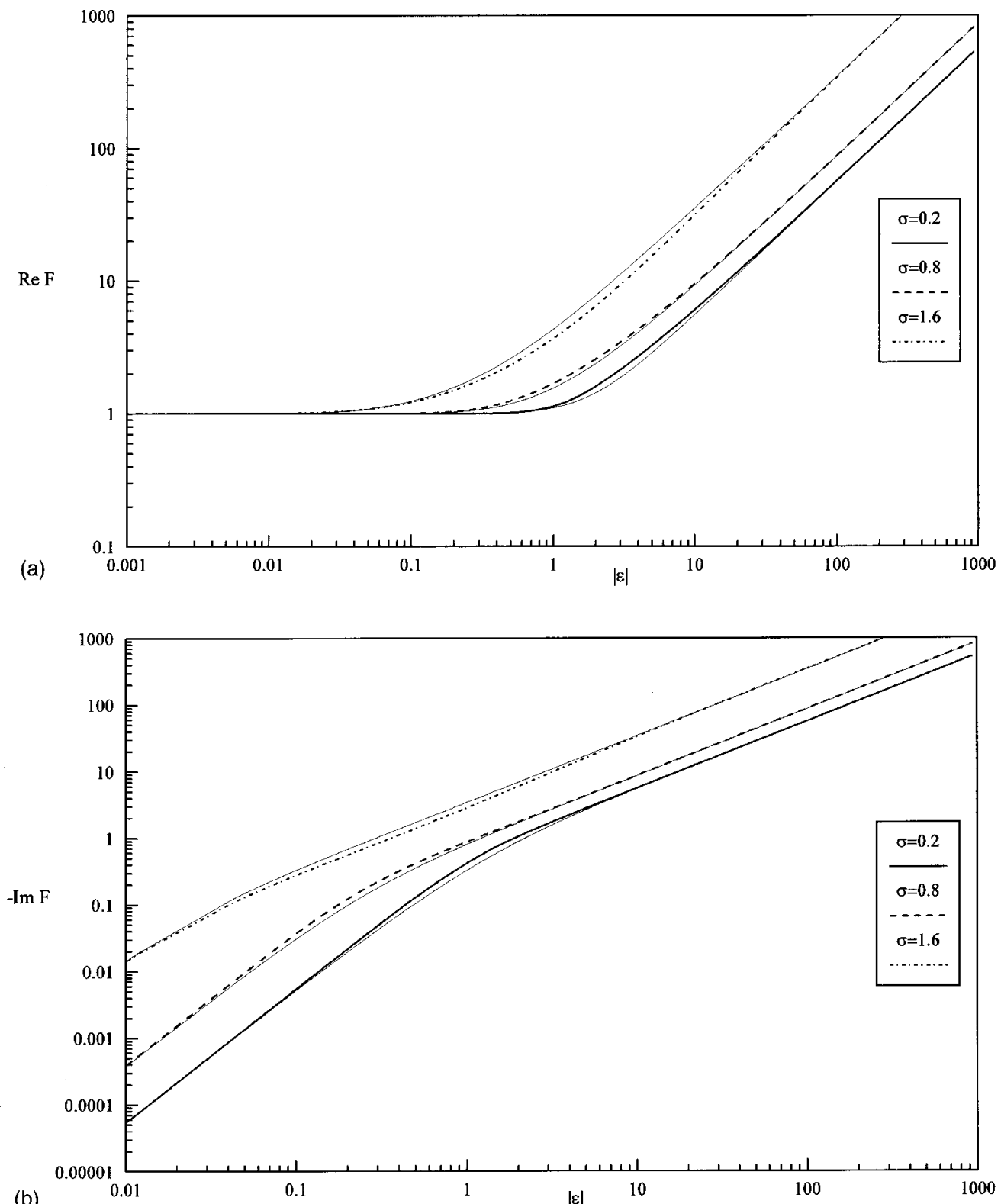

FIG. 2. (a) The real part of the viscosity correction function and its Padé approximant (thin solid lines) in the case of a material with triangular pores. (b) The imaginary part of the viscosity correction function and its Padé approximant (thin solid lines) in the case of a material with triangular pores.

\section{ASYMPTOTIC APPROXIMATIONS FOR LOW AND HIGH FREQUENCY}

For none of the particular forms (38)-(40) for $\chi(z)$ can the integral (34) be evaluated analytically, but approximations valid for low and high values of $\bar{\lambda}$ (corresponding to low and high values of frequency $\omega$, with other variables fixed) can be obtained via expansions of $\chi(z)$ for small and large $z$.

To obtain these approximations using results in the Appendix we write, from (34),

$$
I(\omega) \equiv I(\sqrt{-i} \bar{\lambda}, d),
$$

where, for convenience, we introduce $d=\sigma \ln 2$ and define the function

$$
I(z, d)=\frac{1}{\sqrt{2 \pi}} \int_{-\infty}^{+\infty} \chi\left(z e^{-d t}\right) e^{-t^{2} / 2} d t
$$

for $-\pi / 2<\arg z<\pi / 2$ and $d \geqslant 0$.

Determination of the asymptotic behavior of $\psi$ $=\psi(x, y ; w)$ as $w \rightarrow 0$ is a regular perturbation problem and, for any pore shape, $\psi$ has a power series expansion in $w^{2}$, convergent for small $w$ :

$$
\psi(x, y ; w)=\psi_{0}(x, y)+w^{2} \psi_{1}(x, y)+w^{4} \psi_{2}(x, y)+\cdots .
$$

Substituting this expansion into (3) and comparing powers of $w^{2}$ we find that each of the terms $\psi_{0}, \psi_{1}, \ldots$ satisfies a Poisson's equation, that is,

$$
\Delta \psi_{0}=1, \Delta \psi_{n}=\psi_{n-1}, \quad n=1,2, \ldots
$$

within the pore cross-section (of size $s=1$ ), with $\psi_{n}=0$ on the pore boundary, for $n=0,1, \ldots$ From the maximum principle it follows by induction that $\psi_{n} \leqslant 0$ for $n$ even and that $\psi_{n} \geqslant 0$ for $n$ odd. Thus $\bar{\psi}(z)$ has the power series expansion,

$$
\bar{\psi}(z)=\bar{\psi}_{0}+z^{2} \bar{\psi}_{1}+z^{4} \bar{\psi}_{2}+\cdots,
$$



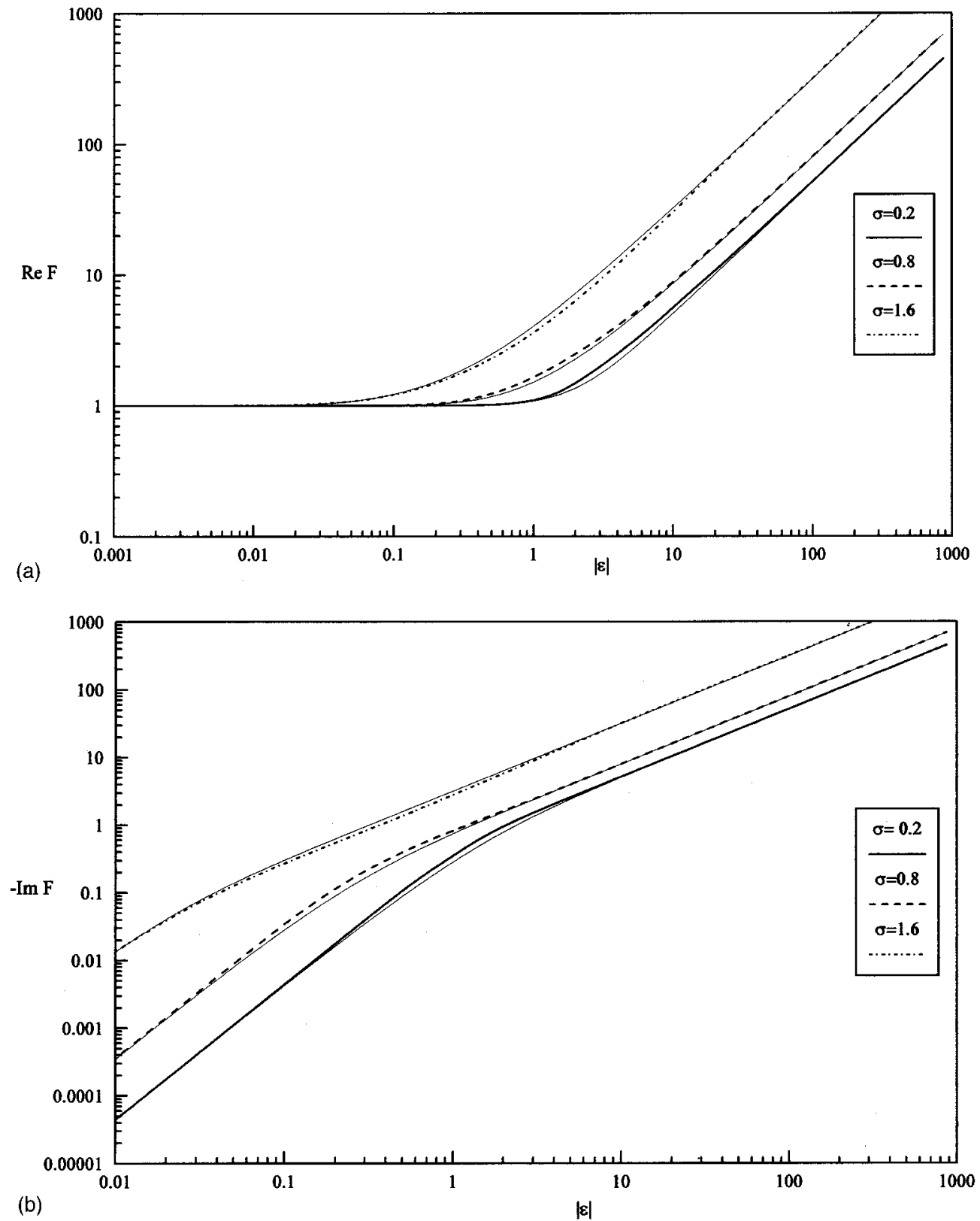

FIG. 3. (a) The real part of the viscosity correction function and its Padé approximant (thin solid lines) in the case of a material with circular cylindrical pores. (b) The imaginary part of the viscosity correction function and its Padé approximant (thin solid lines) in the case of a material with circular cylindrical pores.

convergent for small $z$, with $\bar{\psi}_{n}$ the mean value of $\psi_{n}(x, y)$ over the pore cross-section (of size $s=1$ ) and

$$
\begin{array}{ll}
\bar{\psi}_{n}<0, & n=0,2, \ldots, \\
\bar{\psi}_{n}>0, & n=1,3, \ldots,
\end{array}
$$

so that, for small $z$,

$$
\chi(z)=1+z^{2} \bar{\psi}_{0}+z^{4} \bar{\psi}_{1}+\cdots .
$$

It now follows from (42) and (46), and the results in the Appendix that $I(z, p)$ has the asymptotic expansion

$$
I(z, d) \sim 1+\sum_{n=1}^{\infty} \bar{\psi}_{n-1} z^{2 n} e^{2 n^{2} d^{2}}
$$

as $z \rightarrow 0$, so that

$$
I(\omega) \sim 1+\sum_{n=1}^{\infty} \bar{\psi}_{n-1}(-i)^{n} \bar{\lambda}^{2 n} e^{2 n^{2} d^{2}}
$$

as $\bar{\lambda} \rightarrow 0$ (the low-frequency limit).

Considering now the high-frequency limit; we note that, for $w$ large, $\psi \approx-1 / w^{2}$ across the pore cross-section except within a boundary layer of width $O(1 / w)$. A local analysis of the behavior within this boundary layer yields that (cf. Ref. 9), for a piecewise smooth boundary,

$$
\begin{aligned}
\psi(x, y ; w)= & \frac{1}{w^{2}}\left(e^{-w r}-1\right)+O\left(\frac{1}{w^{2}} e^{-w D}\right) \\
& +O\left(\frac{1}{w^{3}} e^{-w r}\right), \text { as } w \rightarrow \infty
\end{aligned}
$$



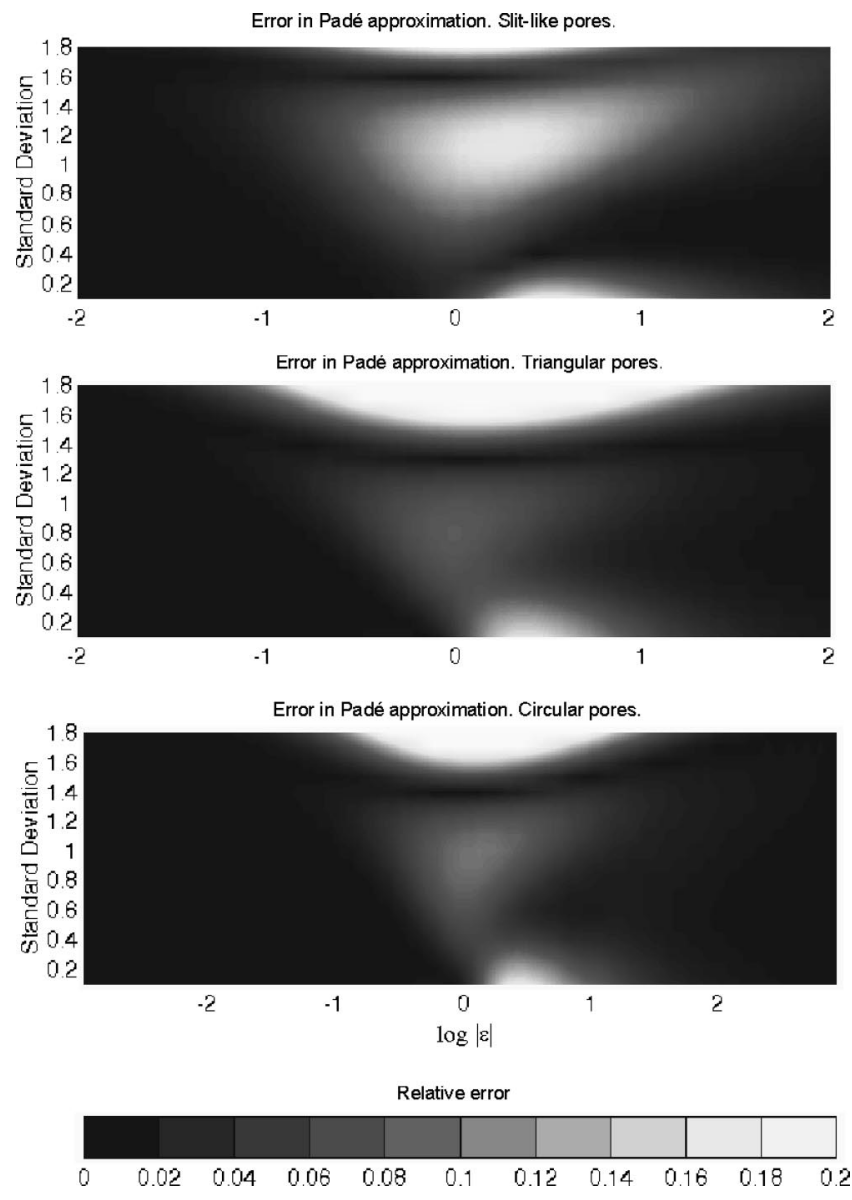

FIG. 4. Distribution of the relative error $\Delta F$ for different values of the standard deviation and dimensionless parameter $|\varepsilon|$.

where $r$ is the distance of $(x, y)$ from the pore boundary and $D$ is the distance to the nearest corner point.

Integrating (49) over the pore cross-section we find that

$$
\bar{\psi}(z)=-\frac{1}{z^{2}}+\frac{A_{1}}{z^{3}}+O\left(\frac{1}{z^{4}}\right), \text { as } z \rightarrow \infty,
$$

where the pore shape factor $A_{1}$ is the ratio of the length of the perimeter to the area of the cross-section for a pore of size $s=1$. It follows that

$$
\chi(z)=\frac{A_{1}}{z}+O\left(\frac{1}{z^{2}}\right), \text { as } z \rightarrow \infty,
$$

and hence, using the results in the Appendix, that

$$
I(z, d)=\frac{A_{1}}{z} e^{d^{2} / 2}+O\left(\frac{1}{z^{2}}\right), \text { as } z \rightarrow \infty,
$$

so that

$$
I(\omega)=\frac{A_{1}}{\sqrt{-i} \bar{\lambda}} e^{d^{2} / 2}+O\left(\frac{1}{\bar{\lambda}^{2}}\right),
$$

as $\bar{\lambda} \rightarrow \infty$ (the high-frequency limit).

The coefficient $A_{1}$ is easily calculated for any pore cross-section. For general pore shapes the coefficients $\bar{\psi}_{0}, \bar{\psi}_{1}, \cdots$ can be obtained via a numerical solution of the Poisson's equations (43). For the particular case of slitlike, circular, and triangular pores these coefficients, which are seen in (46) to be the coefficients in the power series expansion for $\chi(z)$, can be obtained exactly. From (38) to (40), and the power series for tanh $z$ and the modified Bessel functions $I_{0}(z)$ and $I_{1}(z),{ }^{10}$ we have that, for slitlike pores,

$$
\chi(z)=1-\frac{z^{2}}{3}+\frac{2}{15} z^{4}+O\left(z^{6}\right), \quad z \rightarrow 0
$$

for triangular pores,

$$
\chi(z)=1-\frac{z^{2}}{80}+\frac{z^{4}}{4480}+O\left(z^{6}\right), \quad z \rightarrow 0 ;
$$

and for circular pores,

$$
\chi(z)=1-\frac{z^{2}}{8}+\frac{z^{4}}{48}+O\left(z^{6}\right), \quad z \rightarrow 0 .
$$

Thus the coefficients $\bar{\psi}_{0}, \bar{\psi}_{1}$ and $A_{1}$ for these pore shapes are as tabulated in Table I.

For a general pore size distribution, taking the limit $\omega$ $\rightarrow 0$ in (18), it is found that

$$
I(\omega)=1-\frac{i \rho_{0} \omega}{\mu} \bar{\psi}(0)\left\langle s^{2}\right\rangle+o(\omega), \text { as } \omega \rightarrow 0,
$$

where $\left\langle s^{2}\right\rangle=\int_{0}^{\infty} s^{2} e(s) d s$. Thus, taking the limit $\omega \rightarrow 0$ in (17) and noting that $\lim _{\omega \rightarrow 0} F(\omega)=1$ and $\bar{\psi}(0)=\bar{\psi}_{0}$, we

\begin{tabular}{|c|c|c|c|c|c|}
\hline Pore shape & $\bar{\psi}_{0}$ & $\bar{\psi}_{1}$ & $A_{1}$ & $\bar{\psi}_{1} / \bar{\psi}_{0}^{2}$ & $A_{1} \sqrt{-\bar{\psi}_{0}}$ \\
\hline Slit-type & $-\frac{1}{3}$ & $-\frac{2}{15}$ & 1 & $\frac{6}{5}$ & $\frac{1}{\sqrt{3}}$ \\
\hline Triangular & $-\frac{1}{80}$ & $\frac{1}{4480}$ & $4 \sqrt{3}$ & $\frac{10}{7}$ & $\sqrt{\frac{3}{5}}$ \\
\hline Circular & $-\frac{1}{8}$ & $-\frac{1}{48}$ & 2 & $\frac{4}{3}$ & $\frac{1}{\sqrt{2}}$ \\
\hline
\end{tabular}
find that

$$
R_{x}=\frac{-\mu}{\bar{\psi}_{0}\left\langle s^{2}\right\rangle}=\frac{-\mu}{\bar{\psi}_{0} \bar{s}^{2} e^{2 d^{2}}}
$$

in the case of a log-normal pore size distribution.

TABLE I. Values of the coefficients $\bar{\psi}_{0}, \bar{\psi}_{1}, A_{1}$ and the pore shape factors $\bar{\psi}_{1} / \bar{\psi}_{o}^{2}$ and $A_{1} \sqrt{-\bar{\psi}_{o}}$. Note that the numerical values of the coefficients $\bar{\psi}_{0}, \bar{\psi}_{1}, A_{1}$, depend on the definition of $s$, for each pore geometry. The values of the pore shape factors $\bar{\psi}_{1} / \bar{\psi}_{o}^{2}$ and $A_{1} \sqrt{-\bar{\psi}_{o}}$ are independent of this choice. 

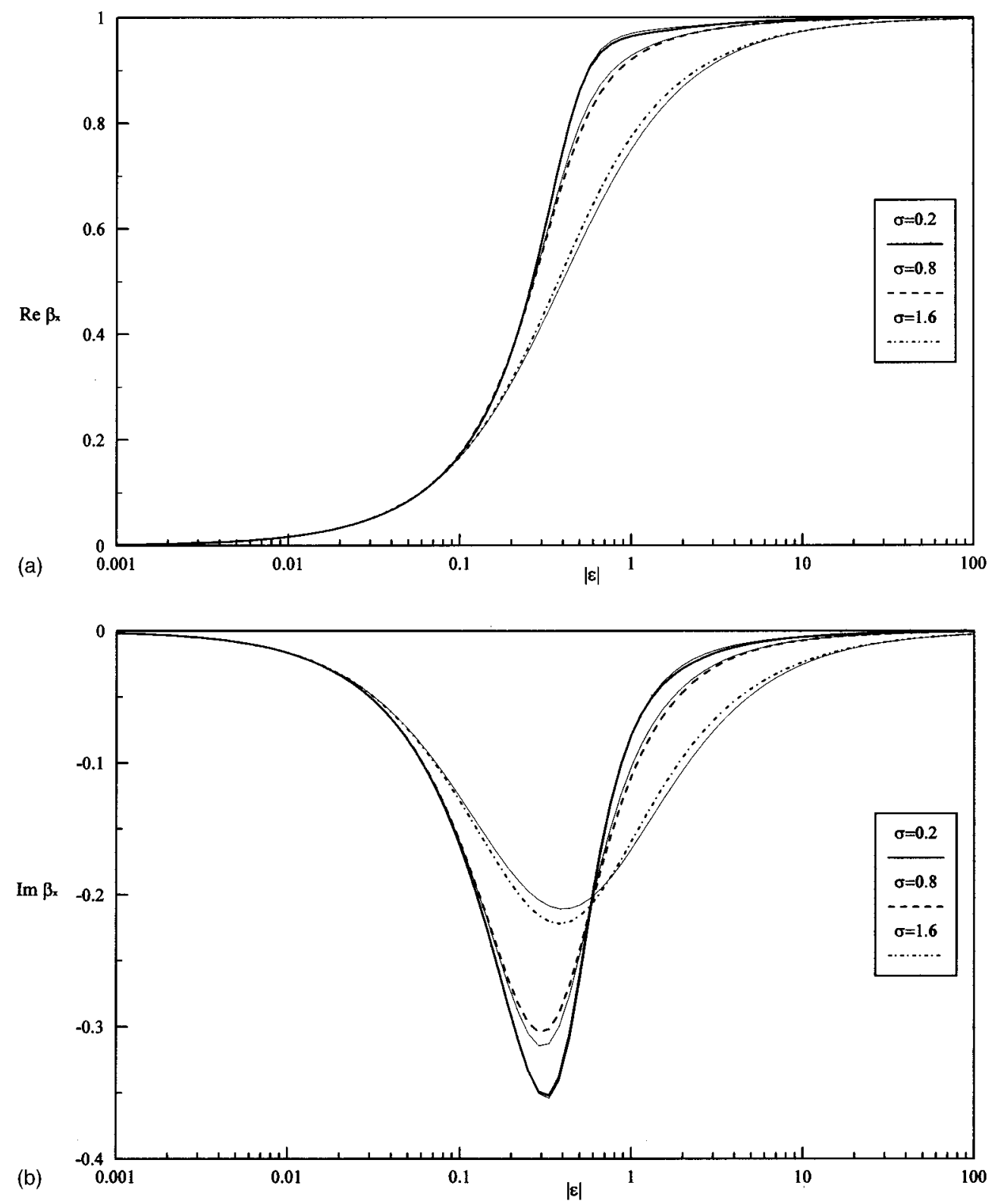

FIG. 5. (a) The real part of the relative admittance and the result obtained via Padé approximation (thin solid lines) for a material with circular cylindrical pores. (b) The imaginary part of the relative admittance and the result obtained via Padé approximation (thin solid lines) for a material with circular cylindrical pores.

Using (51), (17) and (48), it can be shown that, in the low - frequency/high flow resistivity limit $\bar{\lambda} \rightarrow 0$,

$$
\begin{aligned}
F(\omega) & =1+i \bar{\lambda}^{2} e^{2 d^{2}}\left(\frac{\bar{\psi}_{1}}{\bar{\psi}_{0}} e^{4 d^{2}}-\bar{\psi}_{0}\right)+O\left(\bar{\lambda}^{4}\right) \\
& =1+\theta_{1} \varepsilon^{2}+O\left(\varepsilon^{4}\right)
\end{aligned}
$$

where

$$
\theta_{1}=\frac{\bar{\psi}_{1}}{\bar{\psi}_{0}^{2}} e^{4 d^{2}}-1,
$$

and the complex-valued, dimensionless parameter combination $\varepsilon$ is given by

$$
\varepsilon=\sqrt{\frac{-i \omega \rho_{0}}{R_{x}}}=\sqrt{-i \bar{\psi}_{0}} e^{d^{2}} \bar{\lambda}
$$

Similarly, using (51), (17) and (50), we find that, in the highfrequency/low flow resistivity limit $\bar{\lambda} \rightarrow \infty$,

$$
F(\omega)=\sqrt{-i} \bar{\lambda} \bar{\psi}_{0} A_{1} e^{5 d^{2} / 2}+O(1)=\theta_{2} \varepsilon+O(1),
$$

where

$$
\theta_{2}=A_{1} \sqrt{-\bar{\psi}_{0}} e^{3 d^{2} / 2}
$$

For the convenience of the reader the values of the pore shape factors $\bar{\psi}_{1} / \bar{\psi}_{0}^{2}$ and $A_{1} \sqrt{-\bar{\psi}_{0}}$ for slit-type, triangular and circular pores are tabulated in Table I. 


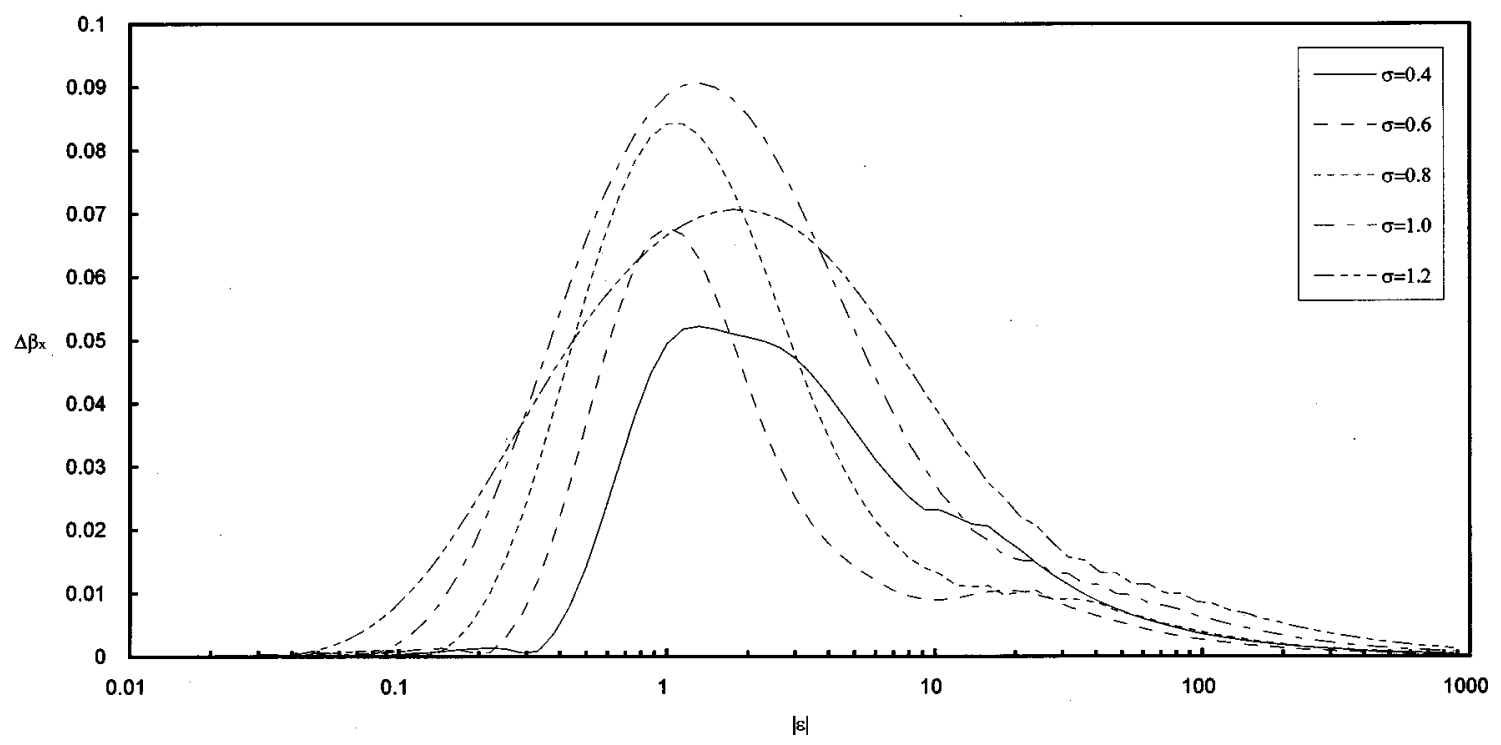

FIG. 6. The relative error in the relative admittance computed for several values of the standard deviation for a medium with circular cylindrical pores.

Utilizing these low- and high-frequency approximations for the viscosity correction function we can obtain the lowand high-frequency behavior of the complex density $\rho_{x}(\omega)$. From (14) it follows that

$$
\frac{\rho_{x}(\omega)}{\rho_{0}}=1+\varepsilon^{-2} F(\omega)
$$

so that, in the low-frequency/high flow resistivity limit, $\varepsilon$ $\rightarrow 0$,

$$
\frac{\rho_{x}(\omega)}{\rho_{0}}=\frac{1}{\varepsilon^{2}}+1+\theta_{1}+O\left(\varepsilon^{2}\right)=\frac{i R_{x}}{\omega \rho_{0}}+\frac{\bar{\psi}_{1}}{\bar{\psi}_{0}^{2}} e^{4 d^{2}}+O\left(\varepsilon^{2}\right) .
$$

In the high-frequency/low flow resistivity limit, $\varepsilon \rightarrow \infty$,

$$
\begin{aligned}
\frac{\rho_{x}(\omega)}{\rho_{0}} & =1+\frac{\theta_{2}}{\varepsilon}+O\left(\varepsilon^{-2}\right) \\
& =1+2 \sqrt{\frac{\mu}{-i \omega \rho_{0}}} \frac{e^{d^{2} / 2}}{\bar{r}_{h}}+O\left(\varepsilon^{-2}\right) .
\end{aligned}
$$

To obtain this last equation we use expression (51) and that $2 \bar{s} / A_{1}=\bar{r}_{h}$, where $\bar{r}_{h}$ is the median hydraulic radius, and the hydraulic radius of a pore is defined as twice the ratio of its cross-sectional area to its perimeter. Equations (57) and (58) agree with those in, e.g., Stinson and Champoux ${ }^{10}$ for the case $d=0$ of uniform pore size.

From (57) and (58) the low- and high-frequency behavior of all acoustic quantities can be deduced using relations (22), (23) and (28).

\section{TWO-POINT PADÉ APPROXIMANTS}

Equations (52) and (55) provide good approximations to the viscosity correction function for small and large values of $|\varepsilon|=\left(\rho_{0} \omega / R_{x}\right)^{1 / 2}$, respectively. To provide an interpolation between these approximations for small and large $|\varepsilon|$ and, hopefully, a sufficiently accurate approximation for intermediate values of $|\varepsilon|$, we construct a two-point Padé approxi- mant, i.e., a rational approximant $\widetilde{F}(\omega)$ which has the same asymptotic behavior as the viscosity correction function $F(\omega)$ at the two points $\varepsilon \rightarrow 0$ and $\varepsilon \rightarrow \infty$. ${ }^{11}$

Let

$$
\widetilde{F}(\omega)=\frac{1+\theta_{3} \varepsilon+\theta_{1} \varepsilon^{2}}{1+\theta_{3} \varepsilon}
$$

where $\theta_{3}=\theta_{1} / \theta_{2}$ and $\theta_{1}$ and $\theta_{2}$ are given, by (53) and (56), in terms of $d=\sigma \ln 2$ and the pore shape factors $\bar{\psi}_{1} / \bar{\psi}_{0}^{2}$ and $A_{1} \sqrt{-\bar{\psi}_{0}}$ (tabulated for various shapes in Table I). Then it is easy to see that $\widetilde{F}(\omega)$ has the required characteristics, i.e., that $\widetilde{F}(\omega)$ has the same asymptotic behavior, (52) and (55), for small and large $|\varepsilon|$, as $F(\omega)$ : precisely

$$
F(\omega)-\widetilde{F}(\omega)=O\left(\varepsilon^{4}\right), \quad \varepsilon \rightarrow 0,
$$

and

$$
F(\omega)-\widetilde{F}(\omega)=O(1), \quad \varepsilon \rightarrow \infty .
$$

Noting that the values of $F(\omega)$ and $\widetilde{F}(\omega)$ depend only on the pore shape and the values of the dimensionless parameter combination $|\varepsilon|$ and the (dimensionless) standard deviation $\sigma$, we now investigate the accuracy of the approximation $\widetilde{F}(\omega)$ for $F(\omega)$ for various pore shapes, various values of $\sigma$, and $0 \leqslant|\varepsilon|<+\infty$.

\section{A. Error in the approximation of the viscosity correction function}

Approximation (59) has been computed for several values of the standard deviation $\sigma$, for all three types of pore geometry, and has been compared with the exact viscosity correction function obtained from Eq. (17), with the integral $I(\omega)$, given by (34), computed by numerical integration.

The real and imaginary parts of the approximation $\widetilde{F}(\omega)$ are compared with those of the exact viscosity correction function $F(\omega)$ in Figs. 1, 2, and 3, for slit-type, triangular, and circular pores, respectively, for $\sigma=0.2,0.8$, and 1.6. As expected, in view of the asymptotic estimates (60) and (61), 

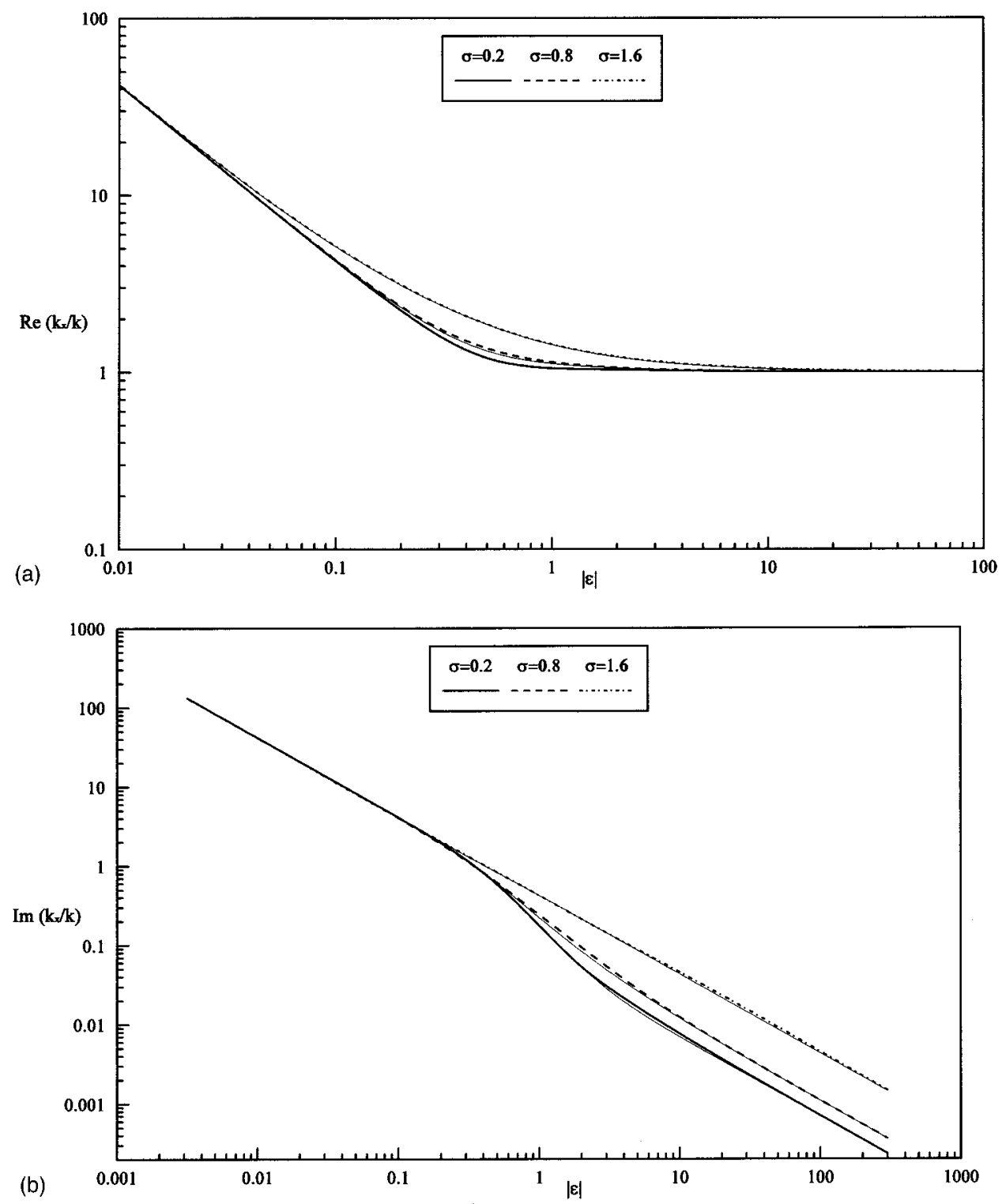

FIG. 7. (a) The real part of the complex wave number and the result obtained via Padé approximation (thin solid lines) for a material with slitlike pores. (b) The imaginary part of the complex wave number and the result obtained via Padé approximation (thin solid lines) for a material with slitlike pores.

the approximation is accurate for $|\varepsilon|=\left(\rho_{0} \omega / R_{x}\right)^{1 / 2}$ small and $|\varepsilon|$ large. The accuracy of approximation deteriorates somewhat in the intermediate range $0.1<\varepsilon<10$.

To quantify this error more precisely, we have computed the magnitude of the relative error, i.e.,

$$
\Delta F(\omega)=\left|\frac{F(\omega)-\widetilde{F}(\omega)}{F(\omega)}\right| .
$$

Figure 4 shows contour plots of $\Delta F(\omega)$ as a function of $\sigma$ and $|\varepsilon|$ for the three pore shapes. It can be seen that the areas of maximum error are mainly concentrated in the range 1 $<|\varepsilon|<10$ and that, as a function of $\sigma$, the error has two distinctive mimima at around $\sigma=0.4$ and $\sigma=1.5$. These graphs can be used for estimating the ranges of $|\varepsilon|$ and $\sigma$ for which $\widetilde{F}(\omega)$ satisfies given accuracy requirements.

Precise calculations indicate that the maximum relative error in the range of interest for most practical applications, $0.2<\sigma<1.5$, does not exceed $15.5 \%(\sigma=1.1)$ for slits, $11 \%$ $(\sigma=1.5)$ for triangles, and $9 \%(\sigma=1.0)$ for a circular pore cross-section.

\section{B. Error in the approximation of other acoustic quantities}

A comparison has also been made between acoustical quantities calculated approximately, by replacing $F(\omega)$ by its approximation $\widetilde{F}(\omega)$, and their exact values, obtained by computing $F(\omega)$ exactly by numerical integration. Considered are the relative admittance and the complex wave number. Figures 5-8 illustrate graphically, for each of these quantities, results for the pore shape for which the approximation error is greatest.

In Fig. 5, for the case of circular cylindrical pores, the relative admittance $\beta_{x}(\omega)=\beta_{b}(\omega) / q$, given by (28), is plotted against $|\varepsilon|$ for $\sigma=0.2,0.8$, and 1.6 , as is the approxima- 


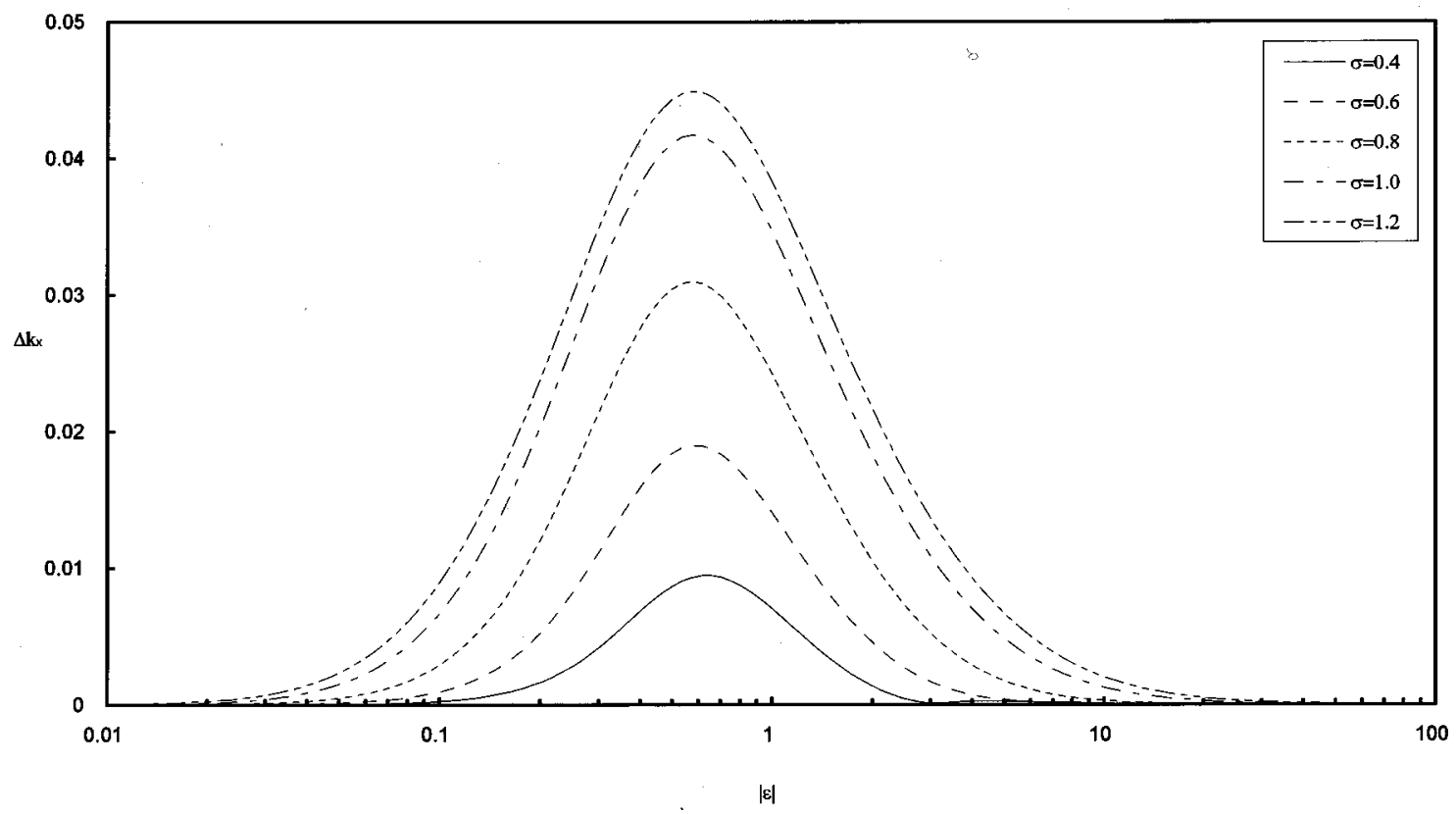

FIG. 8. The relative error in the complex wave number computed for several values of the standard deviation for a medium with slitlike pores.

tion $\widetilde{\beta}_{x}(\omega)$ to $\beta_{x}(\omega)$ obtained by replacing $F(\omega)$ in the computations by its Padé approximant $\widetilde{F}(\omega)$. The agreement is good, especially for $|\varepsilon|$ small and large, and is quantified further in Fig. 6, in which the magnitude of the relative error,

$$
\Delta \beta_{x}(\omega)=\left|\frac{\beta_{x}(\omega)-\widetilde{\beta}_{x}(\omega)}{\beta_{x}(\omega)}\right|
$$

is plotted against $|\varepsilon|$, for various values of $\sigma$. It can be seen that the error in the derived quantity $\beta_{x}(\omega)$ is less than that in the viscosity correction function, and does not exceed $9 \%$ $(\sigma=1.0,|\varepsilon|=1.32)$ for circular pores. Similar calculations show that the maximum error in $\beta_{x}(\omega)$ does not exceed $4 \%$ for slits $(\sigma=1.2,|\varepsilon|=0.44)$, and $2.2 \%$ for triangular pores $(\sigma=1.0,|\varepsilon|=0.38)$.

Analogous trends can be observed for the complex wave number. The approximation to $k_{x}(\omega)$ obtained by replacing $F(\omega)$ by $\widetilde{F}(\omega)$ is denoted by $\widetilde{k}_{x}(\omega)$ and $\Delta k_{x}(\omega)=\mid\left(k_{x}(\omega)\right.$ $\left.-\widetilde{k}_{x}(\omega)\right) / k_{x}(\omega) \mid$ is the relative error. Figure 7 plots the real and imaginary parts of $k_{x}(\omega)$ and $\widetilde{k}_{x}(\omega)$ against $|\varepsilon|$ for $\sigma$ $=0.2,0.8$, and 1.6, and $\Delta k_{x}(\omega)$ is plotted against $|\varepsilon|$ in Fig. 8 . The agreement between both the real and imaginary parts of $k_{x}(\omega)$ and $\tilde{k}_{x}(\omega)$ is excellent. From Fig. 8 and similar graphs we note that the relative error $\Delta k_{x}(\omega)$ does not exceed $4.5 \%(\sigma=1.2,|\varepsilon|=0.58)$ for slitlike pores, $3 \%(\sigma$ $=1.0,|\varepsilon|=0.5)$ for circular pores and $1.4 \%(\sigma=1.2,|\varepsilon|$ $=0.4$ ) for triangular pores.

\section{CONCLUDING REMARKS}

A theoretical justification has been given and simplified expressions presented for the form of the viscosity correction function for a porous medium with a statistically distributed pore size. This function, originally introduced by Biot ${ }^{1}$ and extended by Yamamoto and Turgut ${ }^{3}$ to the case of porous media with a statistically distributed pore size, describes viscous, thermal diffusion and pore size distribution effects of sound propagation inside a porous rigid frame material. Computationally efficient and simple two-point Padé approximants for this function have been derived in this paper for various pore geometries. The resulting equations [(23)(26) and (59)] are simple. The accuracy of the proposed approximation to the exact viscosity correction function has been investigated over the whole range of microscopic material parameters of interest. For most of this range the approximation is highly accurate and it is our belief that the accuracy established over the remaining range will be quite adequate for practical modelling of acoustical characteristics, particularly bearing in mind uncertainties in accurately identifying material parameter values and, indeed, in the accuracy of the model of acoustical characteristics in terms of viscosity correction function proposed.

The simple analytical form of the approximations means that these are likely to be of substantial practical interest for predictions of the acoustic field in the presence of a porous absorbing surface. Some preliminary and encouraging comparisons of excess attenuation predictions, using the proposed approximations for triangular pore shapes, with short range experiments over sand are reported recently in Ref. 12 .

Although the most definite results are only for selected pore geometries, the general equations allow for any complex pore shape to be considered. In this case the shape factors (Table I) which relate to the asymptotic behavior of the solution of the Helmholtz equation for such particular pore shape could be obtained either numerically or experimentally. Specifically, it can be seen from the asymptotic behavior of the viscosity correction function at the lower- and higher-frequency limits [expressions (52) and (55), respectively] that the shape factors, $\bar{\psi}_{1} / \bar{\psi}_{0}^{2}$ and $\sqrt{\bar{\psi}_{0}} A_{1}$, can be recovered from low/high-frequency acoustical experiments on the material combined with nonacoustical measurements of other parameters. 


\section{ACKNOWLEDGMENTS}

The authors would like to thank Dr. S. Taherzadeh for providing FORTRAN code for numerical evaluation of the integrals and Dr. D. C. Hothersall for his comments on this manuscript. This work was part of a project supported by the Engineering and Physical Sciences Research Council under Grant No. GR/H41966.

\section{APPENDIX: APPROXIMATION OF A CLASS OF INTEGRALS}

Suppose that $f(z)$ is a complex-valued function of the complex variable $z$, and that, for some $\alpha$ in the range 0 $<\alpha \leqslant \pi, f(z)$ is continuous in the sector of the complex plane, $|\arg z| \leqslant \alpha,|z|>0$. In Sec. II integrals of the form

$$
I(z, d)=\frac{1}{\sqrt{2 \pi}} \int_{0}^{\infty} f\left(e^{-d t} z\right) e^{-t^{2} / 2} d t
$$

arise, for various choices of the function $f$.

Consider the approximation of $f$ by a function $\tilde{f}$ of the form

$$
\tilde{f}(z)=\sum_{n=1}^{N} a_{n} z^{b_{n}},
$$

where $a_{1}, \cdots, a_{n}, b_{1}, \cdots, b_{n}$ are real coefficients, and suppose that the remainder, $r(z)=f(z)-\widetilde{f}(z)$, satisfies, for some real constants $c$ and $C(C>0)$,

$$
|r(z)| \leqslant C|z|^{c},
$$

for $|\arg z| \leqslant \alpha,|z|>0$. Let $\widetilde{I}(z, d)$ denote the corresponding approximation to $I(z, d)$, i.e.,

$$
\begin{aligned}
\widetilde{I}(z, d) & =\frac{1}{\sqrt{2 \pi}} \int_{-\infty}^{+\infty} \widetilde{f}\left(e^{-d t} z\right) e^{-t^{2} / 2} d t \\
& =\sum_{n=1}^{N} \frac{a_{n} z^{b_{n}}}{\sqrt{2 \pi}} \int_{-\infty}^{+\infty} e^{-d b_{n} t} e^{-t^{2} / 2} d t .
\end{aligned}
$$

Now, for any real number $p$,

$$
\begin{aligned}
\frac{1}{\sqrt{2 \pi}} \int_{-\infty}^{+\infty} e^{-p t} e^{-t^{2} / 2} d t & =\frac{e^{p^{2} / 2}}{\sqrt{2 \pi}} \int_{-\infty}^{+\infty} e^{-(p-t)^{2} / 2} d t \\
& =e^{p^{2} / 2}
\end{aligned}
$$

since

$$
\frac{1}{\sqrt{2 \pi}} \int_{-\infty}^{+\infty} e^{-t^{2} / 2} d t=1
$$

Thus

$$
\widetilde{I}(z, d)=\sum_{n=1}^{N} a_{n} z^{b_{n}} e^{d^{2} b_{n}^{2} / 2} .
$$

Further, the error in this approximation satisfies

$$
I(z, d)-\widetilde{I}(z, d)=\frac{1}{\sqrt{2 \pi}} \int_{-\infty}^{+\infty} r\left(e^{-d t} z\right) e^{-t^{2} / 2} d t
$$

and, utilizing (A2) and (A3) it follows that, for $|\arg z| \leqslant \alpha$,

$$
\begin{aligned}
|I(z, d)-\widetilde{I}(z, d)| & \leqslant \frac{C}{\sqrt{2 \pi}}|z|^{c} \int_{-\infty}^{+\infty} e^{-c d t} e^{-t^{2} / 2} d t \\
& =C|z|^{c} e^{c^{2} d^{2} / 2} .
\end{aligned}
$$

${ }^{1}$ M. A. Biot, "Theory of propagation of elastic waves in a fluid-saturated porous solid. I. Low-Frequency Range,' J. Acoust. Soc. Am. 28, 168178 (1956); “II. Higher Frequency Range,' J. Acoust. Soc. Am. 28, 179-191 (1956)

${ }^{2}$ M. R. Stinson, "The propagation of plane sound waves in narrow and wide circular tubes, and generalisation to uniform tubes of arbitrary crosssectional shape,', J. Acoust. Soc. Am. 89, 550-558 (1991).

${ }^{3} \mathrm{~T}$. Yamamoto and A. Turgut, "Acoustic-wave propagation through porous-media with arbitrary pore-size distributions," J. Acoust. Soc. Am. 83, 1744-1751 (1988).

${ }^{4}$ Keith Attenborough, "Models for the acoustical properties of air-saturated granular media,', Acta Acust. (China) 1, 213-226 (1993).

${ }^{5}$ S. N. Chandler-Wilde and K. V. Horoshenkov, "Padé approximants for the acoustical characteristics of rigid frame porous media,' J. Acoust. Soc. Am. 98, 1119-1129 (1995).

${ }^{6} \mathrm{~J}$. M. Evans, "Measuring the pore size distribution of a model granular material using a water suction method,' Working paper/Internal report, Department of Engineering Mechanics, Open University, October 1993.

${ }^{7}$ C. H. Juang and R. D. Holtz, "Fabric, pore size distribution, and permeability of sandy soils,' J. Geotechn. Eng. Am. Soc. Civil Eng. 112, 855868 (1986).

${ }^{8}$ K. V. Horoshenkov, D. C. Hothersall, and K. Attenborough, "Porous materials for scale model experiments in outdoor sound propagation,' J. Sound Vib. 194, 685-708 (1996).

${ }^{9}$ M. R. Stinson and Y. Champoux, "Propagation of sound and the assignment of shape factors to model porous materials having simple pore shape geometries,', J. Acoust. Soc. Am. 91, 685-695 (1992).

${ }^{10}$ M. Abramovitz and I. A. Stegun, Handbook of Mathematical Functions (Dover, New York).

${ }^{11}$ G. A. Backer, Essentials of Padé Approximants (Academic, London, 1975).

${ }^{12}$ K. Attenborough, "Natural noise control,' Proceedings of International Congress on Noise Control Engineering, pp. 51-73, Liverpool, England, July-August 1996. 